\title{
Comprehensive Effect of Salt Stress and Peanut Cultivars on the Bacterial Community Structure and Diversity of Peanut Rhizosphere Soils
}

\section{Yang $\mathrm{Xu}$}

Shandong Peanut Research Institute

Hong Ding

Shandong Peanut Research Institute

Saiqun Wen

Shandong Peanut Research Institute

Dunwei Ci

Shandong Peanut Research Institute

\section{Guanchu Zhang}

Shandong Peanut Research Institute

\section{Guang Yuan}

Shandong Peanut Research Institute

\section{Feifei Qin}

Shandong Peanut Research Institute

\section{Liangxiang Dai}

Shandong Peanut Research Institute

Zhimeng Zhang ( $\sim$ qinhdao@163.com )

Shandong Peanut Research Institute, Shandong Academy of Agricultural Sciences, Qingdao, Shandong, 266100, P. R. China https://orcid.org/0000-0001-8975-5503

\section{Research article}

Keywords: 16S rRNA, Rhizosphere bacterial community, Peanut cultivars, Salt stress, Arachis hypogaea L

Posted Date: August 13th, 2020

DOl: https://doi.org/10.21203/rs.3.rs-55891/v1

License: (c) (1) This work is licensed under a Creative Commons Attribution 4.0 International License.

Read Full License 


\section{Abstract}

Background: Plant rhizosphere bacterial communities influence plant growth and stress tolerance, which differs across cultivars and external environments. Peanut (Arachis hypogaea. L) as an important oil crop cultivated worldwide. However, relatively little is known about the comprehensive effects of environmental conditions and peanut cultivars on rhizosphere bacterial community structure and diversity.

Results: Here, bacterial community structure diversity from rhizosphere soils of various susceptible and resistant peanut cultivars with or without salt stress was analyzed by 16S rRNA gene deep sequencing and quantitative PCR assays. Taxonomic analysis showed that the bacterial community predominantly consisted of phyla Actinobacteria, Proteobacteria, Chloroflexi, Acidobacteria, and Cyanobacteria. Among these bacteria, numbers of beneficial bacteria Cyanobacteria and Proteobacteria increased, while that of Acidobacteria decreased after salt treatment. Metabolic function prediction showed that the percentages of reads categorized to signaling transduction and inorganic ion transport and metabolism were higher in the salt-treated soils, which may be beneficial to plant survival and salt tolerance.

Conclusions: Overall, rhizosphere bacterial community structure and population metabolism are affected by salt stress, which may be conducive to peanut stress tolerance in saline-alkali soil. The study is therefore crucially important to develop the foundation for improving the salt tolerance of peanuts via modifying the soil bacterial community.

\section{Background}

Peanut (Arachis hypogaea L.) is a globally important oil and cash crop containing $36-54 \%$ oil, $16-36 \%$ protein, and $10-20 \%$ carbohydrates [1]. China is a major peanut producing, consumption, and export trade country with strategic importance in the development of agriculture and the national economy. Due to the limited arable land and requirement for developing regional agriculture, peanuts have been tried for cultivating in saline-alkali soil of northern China, such as the Yellow River delta coastal areas [2]. Extreme conditions in saline-alkali soil severely affect peanuts' growth and productivity and cause significant economic losses. Exploring salt response mechanisms and further enhancing peanut salt tolerance has become a decisive factor to improve peanut production in the peanut industry.

Plants have evolved complex molecular strategies to withstand salt stress, including the modulation of stress-response genes, accumulation of osmotic substances, activation of antioxidant enzymes, and regulation of terminal $\mathrm{Na}^{+}$determinants (such as Salt Overly Sensitive 1, High-affınity $\mathrm{K}^{+}$transporter 1 , and $\mathrm{Na}^{+} / \mathrm{H}^{+}$antiporter) [3-6]. Additionally, the root external environment has also been reported to influence plant salt tolerance [7-9]. However, extant results in peanut, especially different peanut cultivars are relatively few.

Roots can exude a variety of chemicals and nutrients into the soil surrounding the roots, and then attract various microbes, such as bacteria, algae, fungi, and protozoa living with plant roots $[10,11]$. The nearby 
narrow soil is designated as the rhizosphere, which is a "hot spot" of intense microbial activity [11]. Rhizosphere-associated microbes are active to involve nutrient and water acquisition, decompose organic matter, and exert different kinds of positive effects on plant growth $[12,13]$. The quantity and quality of rhizosphere microbial communities change with the environment due to microbes that can sense environmental signal molecules to allow some specific microbes to increase or decrease $[7,14]$. In the last decade, a variety of studies have emerged involving members of the stress-response plant growth promoting rhizobacteria (PGPRs) to be beneficial to stress tolerance and plant growth through diverse mechanisms such as provide a buffer zone for plants to cope with stresses, alter the nutrient mineralization and soil fertility, produce hormones that promote plant growth or induce systemic resistance for plants [15-18]. Thus, rhizosphere-associated microbes may be beneficial to plant survival and adaptation to environmental stresses. Therefore, studying the colonization and propagation of microbial community and further selecting the stress-tolerance beneficial microbes is very important.

The rhizosphere microbial communities may differ across different cultivars due to the root exudates from some specific cultivars that may inhibit soil microbes by releasing allelochemicals, while others promote the growth of some microbes $[19,20]$. Peanut-associated bacterial community structure may be unique, whereas the study about peanut bacterial community structure and the relationship with salt stress and peanut cultivars have been largely unexplored. In this study, 16S rRNA gene sequencing and quantitative PCR (qPCR) assays were used to compare the independent replicates of rhizosphere soils from four peanut cultivars with or without salt treatment. The study aims to explore the relationships between the changes of soil bacterial community with their related peanut cultivars and salt stress treatment, to provide theoretical basis and practical guidance for the rational planting of peanuts and maintaining soil biodiversity in saline-alkali land.

\section{Results}

\section{Overall and alpha diversity analysis of sequencing data}

Bacterial community structure diversity of rhizosphere soils from four peanut cultivars with or without salt stress was carried out by $16 \mathrm{~S}$ rRNA gene sequencing. Salt-resistant (big-grain peanut Huayu25, small-grain peanut Luhua12) and susceptible (big-grain peanut Huayu33, small-grain peanut Huayu20) peanut cultivars were used in this study. Bulk soil group without plants was collected and designated as control bulk soil (CB). We combined the controlled rhizosphere soil groups from Huayu25, Luhua12, Huayu33, and Huayu20 to controlled rhizosphere soil of resistant big-grain peanut (CRBR), resistant small-grain peanut (CRSR), susceptible big-grain peanut (CSBR), and susceptible small-grain peanut (CSSR), respectively. And salt-treated rhizosphere soil groups from Huayu25, Luhua12, Huayu33, and Huayu20 were designated as salt-treated rhizosphere of resistant big-grain peanut (SRBR), resistant small-grain peanut (SRSR), susceptible big-grain peanut (SSBR), and susceptible small-grain peanut (SSSR), respectively. After filtering using the QIIME software (Quantitative Insights into Microbial Ecology, v1.8.0), 1,357,465 sequences passed quality screening and most of which were between 401 and 420 bp 
(Fig. 1A and Table S1). Operational taxonomic units (OTUs) were generated with a $97 \%$ threshold identity, and a total of 6,429 OTUs were detected in peanut rhizosphere soils (Table S1).

To analyze community richness and diversity, alpha diversity analysis was performed. Alpha diversity indices (Sobs, Shannon and Simpson, Chao1 and ace, and coverage) which can reflect the community richness and diversity of the bacterial community were also performed (Fig. 1B and Table S2). The Good's coverage index is $0.997 \pm 0.001$, indicating an adequate sequencing depth, which is further proved by other alpha diversity analysis. Rarefaction curve analysis showed that in the process of population sampling, the rate of increase of new species increased with the increase of sample size and then gradually leveled off, indicating that the sequencing depth of this peanut rhizosphere soil was high enough to analyze the community richness (Fig. 1C). Rank abundance curve showed that all the rhizosphere soil samples had high species evenness and homogeneity (Fig. 1D). All the results showed that alpha diversity revealed no significant difference among the different soil samples of the peanut rhizosphere.

\section{Peanut Rhizosphere Bacterial Community Structure Taxonomic Analysis}

To further analyze the bacterial community structure, we analyzed the abundance distributions of each soil group at five levels (phylum, classes, orders, families, and genus). The bacterial community of peanut is mainly composed of Actinobacteria, Proteobacteria, Chloroflexi, Acidobacteria, and Cyanobacteria at the phylum level, accounted for more than $80 \%$ of total bacterial sequences (Fig. 2A). Further structure analysis showed that bacterial community abundance indeed altered with salt stress, whereas no significant differences were detected among the four peanut cultivars. Cyanobacteria and Proteobacteria increased in most salt-treated soils, while Acidobacteria decreased in all the salt-treated soils compared to the controlled rhizosphere soils. Among them, the abundance of Cyanobacteria in SRBR slightly higher than that of controlled rhizosphere soils, while lower than that of other salt-treated rhizosphere soils (Fig. 2A and Fig. S1). Furthermore, Chloroflexi were the dominant bacteria in the bulk soils, which decreased in peanut rhizosphere soils (Fig. 2A and Fig. S1). At the class level, most of the bacterial community belonged to Actinobacteria, Alphaproteobacteria, Acidobacteria, Cyanobacteria, and Betaproteobacteria (Fig. 2B). The presence of Acidobacteria and Betaproteobacteria decreased in salttreated soils, whereas numbers of Alphaproteobacteria and Cyanobacteria increased in these soils. Gammaphaproteobacteria increased in all peanut rhizosphere soils than in the bulk soils (Fig. 2B). Beneficial bacteria Rhizobiales were the most abundant order in peanut rhizosphere soils and Micrococcaceae predominated in peanut rhizosphere soils at the family level (Fig. 2C, D).

A thorough investigation at the genus level was performed in our study and the top 10 most abundant genera were shown by Wilcoxon rank-sum test in Fig. 3A. Pseudarthrobacter, norank_c_Acidobacteria, Sphingomonas, and norank_c_Cyanobacteria were predominantly found in the peanut rhizosphere soils. Many sequences named as "norank" or "unclassified" were unidentified species but were abundant in 
peanut rhizosphere soils, demonstrating that the peanut soils remained a challenging reservoir of biodiversity which needed to further study (Fig. 3A and Excel S1). Salt treatment increased the quantities of Rhizobium but reduced the numbers of RB41, Gaiella, norank_o_Gaiellales, and norank_c_Acidobacteria (Fig. 3A). Pie chart analysis was also performed to provide the comprehensible depiction of bacterial groups present in four peanut cultivars with or without salt stress. The top 10 most abundant genera were identical in different rhizosphere soils, whereas the proportions and abundance were significantly different (Fig. 3B and Fig. S2). Thus, salt stress can rapidly cause the shift of bacterial abundance distributions and the enrichment of some specific bacterial species in peanut rhizosphere soils.

\section{Clustering Analysis Of Bacterial Community}

Beta diversity analysis and clustering analysis were performed to check the similarities and dissimilarities among different soil groups. Principal component analysis (PCA) and principal co-ordinates analysis (PCoA) analysis showed that the bacterial community compositions of different soil groups were diverse (Fig. 4A, B). And bacterial community compositions of four salt-treated soil groups were more similar to each other but farther apart from the four controlled rhizosphere soil groups in different coordinates (Fig. 4B). Cluster analysis in the form of a hierarchical tree showed that the bacterial community structures of salt-treated and controlled rhizosphere soils were diverse, whereas the duplicate samples in the same soil group were similar to each other as other beta diversity analyses (Fig. 4C).

Pseudarthrobacte were the most abundant genera in the peanut rhizosphere and $35 \%$ of the top 20 richest genera belonged to the phylum Actinobacteria in the phylogenetic tree (Fig. 5A and Fig. S3). Heat map analysis showed that Flavobacterium, Devosia, and Rhizobium at the genus level occurred relatively higher abundance in salt-treated rhizosphere soils (Fig. 5B). Thus, all the results show that salt stress leads to the change of bacterial community and enrichment of Flavobacterium, Flavobacterium, and Rhizobium at the genus level, which may be beneficial to peanut survival and salt resistance as other PGPRs.

\section{Specific phylotypes of rhizosphere bacterial community modulated by salt stress}

Linear discriminant analysis (LDA) effect size (LEfSe) was employed to identify specific phylotypes of peanut rhizosphere bacterial community responding to salt stress from phylum to genus level in cladograms (Fig. 6 and Fig. S4). LEfSe analysis confirmed the significant enrichment of Rhizobiales (from order to genus) and Flavobacteriia (from class to genus) in the salt-treated group (SSBR) and enrichment of Chloroflexi (from phylum to order) in the bulk soil (CB) found by community structure taxonomic analysis (Figs. 2, 3, and 6). In the bulk soil (CB), additionally found the enrichment of Firmicutes (from phylum to genus, namely p_Firmicutes, c_Bacilli, o_Bacillales, f_Bacillaceae, and g_Bacillus) and TK10 (from class to genus, C_TK10, o_norank_c_TK10, f_norank_c__TK10, and g_norank_c_TK10). In peanut rhizosphere soils of different treatments, the predominant bacterial community was diverse. In controlled rhizosphere soils, phylum Acidobacteria dominated in CRBR, and 
phylum Gemmatimonadetes and Saccharibacteria dominated in CSSR, while class Acidimicrobiales and Clostridiales specifically elevated in CRSR and CSBR, respectively (Fig. 6). After salt stress treatment, Rhodobacterales and Xanthomonadales dominated in SRBR, and phylum Cyanobacteria and genus Sphingomonas were predominant in SRSR (Fig. 6). Additionally, in rhizosphere soils of salt susceptible peanut cultivars, SubsectionIII (from order to genus, namely o_SubsectionIII, f_Familyl_o_SubsectionIII, and g_unclassified_f_FamilyI_o_SubsectionIII) and family Intrasporangiaceae were relatively abundant in SSSR. The bacterial community structure shows significant specificity in different treatments, which may be associated with their survival abilities or potential roles under salt stress.

\section{Metabolic Functional Features Prediction Of Peanut Rhizosphere Bacterial Community}

We then predict the function of bacterial population metabolism based on the known microbial genomic data. The cluster of orthologous groups (COG) database analysis showed that four metabolic functions (namely amino acid transport and metabolism, lipid transport and metabolism, energy production and conversion, and posttranslational modification, protein turnover, chaperones) were more abundant in salttreated soil groups (Figs. 7A, B and Fig. S5). Furthermore, salt stress-related classifications including signal transduction mechanism and inorganic ion transport and metabolism were also enriched in these salt-treated soils (Fig. 7A). These vigorous metabolic functions may be associated with salt stress tolerance of bacterial communities in the salt-treated soils.

\section{Qpcr Of Specific Bacterial Groups}

To further verify the $16 \mathrm{~S}$ rRNA gene sequencing data, qPCR assay further confirmed the changes in the abundance of the major bacterial phyla (Actinobacteria, Proteobacteria, Chloroflexi, Acidobacteria, and Cyanobacteria) and salt-induced bacteria Rhizobium in all soil samples. The numbers of phylum Cyanobacteria and Alphaproteobacteria and beneficial bacteria Rhizobium were significantly higher in salt-treated peanut rhizosphere soils, whereas Acidobacteria was relatively lower in these soil samples as the taxonomic analysis in Fig. 2A (Fig. 8). And Chloroflexi were enriched in the bulk soils, and gradually decreased in peanut rhizosphere soils (Fig. 2A and 8D). Most of the results were consistent with the 16S rRNA gene sequencing analysis. Taken together, all the results indicate that Cyanobacteria and Alphaproteobacteria as predominant phyla in salt-treated soils.

\section{Discussion}

Rhizosphere soil is a very complex ecological system, a wide range of host and environmental factors have been shown to influence plant microbial community compositions [21-23]. In the current study, $16 \mathrm{~s}$ rRNA gene sequencing was used to examine the rhizosphere bacterial community of four peanut cultivars with or without salt stress. Actinobacteria, Proteobacteria, Chloroflexi, Acidobacteria, and Cyanobacteria 
were identified in all the rhizosphere soils in high abundance as similarly reported elsewhere [24, 25]. Further bacterial community structure analysis and qPCR assays showed that salt stress can rapidly lead to the change of bacterial abundance distributions and enrichment of some specific bacterial species in peanut rhizosphere soils, whereas different peanut cultivars only slightly change the bacterial community structure (Fig. 2-5). Cyanobacteria and Proteobacteria dramatically increased in salt-treated soils as dominant bacteria, while Acidobacteria decreased in these soils compared to the controlled soils (Fig. 2A). Cyanobacteria can improve soil environments via accumulating soil carbon and nitrogen, which can survive in harsh environments such as saline-alkali and arid soil $[26,27]$. Proteobacteria is the predominant phylum in the rhizosphere of most plant species may due to their rapid growth rates [28]. In addition, Proteobacteria as beneficial bacteria can degrade aliphatic and aromatic compounds into simpler forms, and survive in some extreme conditions [29]. This work revealed that the increased Cyanobacteria and Proteobacteria under salt stress in our study may be conducive to salt stress tolerance.

The clustering analyses revealed that Flavobacterium, Devosia, and Rhizobium were relatively abundant in salt-treated peanut rhizosphere compared with that of the control (Fig. 5). Flavobacterium is a kind of copiotrophic and fast-growth bacterium with a transient nutrition niche [30]. Rhizobium as a nitrogenfixing bacterium is closely related to peanut nodulation [31]. Devosia has been found to have functions of nitrogen fixation, which can catalyze the conversion of $\mathrm{N}_{2}$ gas to ammonia [32]. The enrichments of these nitrogen-fixing bacteria in peanut rhizosphere soils have important environmental significance for improving soil quality and ensuring plant survival in adverse conditions.

Soil bacterial community plays a vital role in sustaining the basic functions of plants under salt stress. Metabolic functional feature prediction showed that the percentages of reads categorized to defense mechanisms (posttranslational modification, protein turnover, and chaperones and signal transduction mechanism) and biological metabolisms (amino acid transport and metabolism, lipid transport and metabolism, energy production and conversion and chaperones and inorganic ion transport and metabolism) were enriched in the salt-treated soils than that of the controlled soils (Fig. 7A). Amino acids such as glutamine and proline can improve salt tolerance in plants [33,34], and some chaperones, such as heat shock proteins, are also identified as stress tolerance enhancers for plants [23]. Moreover, inorganic ion transport and metabolism is high. Soil salinity can cause excessive ion toxicity $\left(\mathrm{Na}^{+}\right.$and $\mathrm{Cl}^{-}$) to plants or microbes [35], thus the vigorous inorganic ion transport and metabolism in salt-treated soils may have implications for plants to re-establish ionic homeostasis. Therefore, we assume that these vigorous pathways may contribute to peanut adaption to salt stress. This study aims to evaluate and provide some new insight into the effect of peanut cultivars and soil salinity on the bacterial abundance distributions of rhizosphere soil. It may be a good strategy to screen dominant and beneficial soil bacteria and deliver high populations of some desired bacteria around the rhizosphere soil to improve salt tolerance of peanuts.

\section{Conclusions}


In the current work, the study of rhizosphere microbial community of four peanut cultivars under salt and normal conditions via 16S rRNA gene deep sequencing was performed, we found that Actinobacteria, Proteobacteria, Chloroflexi, Acidobacteria, and Cyanobacteria were predominant in all the peanut rhizosphere soils. Salt stress can quickly lead to the shift of microbial community structure and enrichment of Cyanobacteria and Proteobacteria, while Acidobacteria decreased in salt-treated soils compared to the controlled soils. Identifying dominant and beneficial soil bacteria and improving salt tolerance of peanuts via modification of the soil microbes is promising in the future.

\section{Methods}

\section{Plant materials and salt stress treatment}

Salt-susceptible (Huayu33, Huayu20) and salt-resistant (Huayu25, Luhua12) peanut cultivars cultivated in an acrylic tank containing the same weight of topsoil in the greenhouse at Laixi experimental station, China $\left(120.53^{\circ} \mathrm{E}, 36.86^{\circ} \mathrm{N}\right)$. Huayu33 and Huayu25 were big-grain peanut cultivars (hundred-grain weight $95.9 \mathrm{~g}$ and $98 \mathrm{~g}$, respectively), and Huayu20 and Luhua12 were small-grain peanut cultivars (hundredgrain weight $68.6 \mathrm{~g}$ and $70 \mathrm{~g}$, respectively). The topsoil was taken from a peanut field at the Laixi experimental station. When the soil was dry, sieved it with a $1 \mathrm{~cm}$ sieve and added $18 \mathrm{~kg}$ soil to each tank. The physiochemical properties of soil: $\mathrm{pH} 7.7$, EC $0.26 \mathrm{dS} / \mathrm{m}$, total nitrogen $1.70 \mathrm{~g} \cdot \mathrm{kg}^{-1}$, organic content $13.23 \mathrm{~g} \cdot \mathrm{kg}^{-1}$, available potassium $103.20 \mathrm{mg} \cdot \mathrm{kg}^{-1}$, and available phosphorous $11.70 \mathrm{mg} \cdot \mathrm{kg}^{-1}$. For the salt-treated soil group, the required amount of $\mathrm{NaCl}$ was added into the soil to attain to $2.00 \mathrm{~g} \cdot \mathrm{kg}^{-1} \mathrm{salt}$ concentration. Then four fine peanut seeds of different peanut cultivars were planted in each tank, respectively. The water was added every two days to keep the soil water content at $85 \%$ of field capacity (water is kept in the capillary pores of the soil without exudation at $85 \%$ of field capacity, and salt cannot be lost with water to maintain the $2.00 \mathrm{~g} \cdot \mathrm{kg}^{-1}$ soil salt concentration during the whole growth period of the peanut) until the harvest time at $25 \pm 2{ }^{\circ} \mathrm{C}$ and $16 / 8 \mathrm{~h}$ light/dark photoperiod [23]. The experiment was carried out during the rainy season 2019 and sowing was done on 1st May, and harvest was done on 15th September. Plants and soil samples were collected during the harvest time.

\section{The sample collection of rhizosphere compartments for 16S rRNA gene sequencing}

Rhizosphere compartments consisted of root surface soils and soils around the roots [7]. Soils around the roots were obtained by slightly shaking. Then roots were cut off and placed in a centrifuge tube containing PBS buffer ( $0.25 \mathrm{~g}$ of $\mathrm{NaH}_{2} \mathrm{PO}_{4} \cdot \mathrm{H}_{2} \mathrm{O}, 0.66 \mathrm{~g}$ of $\mathrm{Na}_{2} \mathrm{HPO}_{4} .7 \mathrm{H}_{2} \mathrm{O}, 8 \mathrm{ml}$ Silwet L-77, pH 7.0). Highspeed centrifugation of the centrifuge tubes to remove plant debris, and the supernatant was centrifuged at $5000 \times \mathrm{g}$ for $15 \mathrm{~min}$ and the pellets were re-suspend by the PBS buffer to obtain the root surface soil [9]. Each treatment contained three biological replicates and a total of nine soil groups were collected. The bacterial DNA was extracted by using PowerSoil ${ }^{\circledR}$ DNA Isolation Kit (MoBio Laboratories, Carlsbad, $\mathrm{CA})$. 


\section{6s Rrna Gene Sequencing}

The quality and concentrations of extracted DNA were checked before being used for bacterial 16S rRNA gene amplification by Majorbio (Shanghai, China) [7]. The V3 and V4 region of the 16S rRNA gene was amplified using the specific primers 338F (forward primer, 5'-ACTCCTACGGGAGGCAGCAG-3') and 806R (reverse primer, 5'-GGACTACHVGGGTWTCTAAT-3'). 2 × Phanta Max Master Mix (P515, Vazyme, Nanjing, China) was used in PCR reactions and then the Tiangen Gel Extraction Kit (Tiangen, Beijing, China) was used to purify the PCR product [36]. The sequencing libraries were obtained by using TruSeq ${ }^{\circledR}$ DNA PCRFree Sample Preparation Kit (Illumina, San Diego, CA, USA) and 250 bp/300 bp paired-end reads were generated. Reads were submitted to National Center for Biotechnology Information (NCBI) Sequence Read Archive (SRA) under accession SUB6650557 and bioproject PRJNA592826, database is accessible via the link https://www.ncbi.nlm.nih.gov/sra/PRJNA592826.

\section{Otu Cluster And Bioinformatic Analysis}

Paired-end reads were merged using FLASH (version 1.2.7) and raw tags were preliminarily screened according to the QIIME (Quantitative Insights into Microbial Ecology, v1.8.0) quality-controlled process [37]. Chimera sequences were detected and deleted via compared with the reference Gold database (http://drive5.com/uchime/uchime_download.html) using UCHIME algorithm [38]. The operational taxonomic unit (OTU) was defined as the sequence that the similarity threshold set $\geq 97 \%$ [39]. The gplot package of $\mathrm{R}$ software was used to cluster the community composition data according to the abundance distribution of taxon or the similarity between soil groups to generate heat maps [40].

\section{Alpha And Beta Diversity Analysis}

In order to compute alpha diversity, we calculated six indices: both Chao1 and ace estimate the species abundance; Sobs exhibits OTU numbers; Shannon and Simpson exhibit species diversity; Coverage can reflect sequencing depth. Rarefaction curves were generated to evaluate the species richness and sequence depth. The rank abundance curve reflects the species abundance and evenness [41].

The similarity or dissimilarities of the community structure between soil samples was analyzed by beta diversity analysis. The similarities or dissimilarities among 9 soil groups were studied by using principal component analysis (PCA), principal co-ordinates analysis (PCoA), and clustering analysis in the form of a hierarchical tree [42].

Linear discriminant analysis (LDA) effect size (LEfSe) and the cluster of orthologous groups (COG) database analysis

LEfSe is a high dimensional biomarker algorithm for the analysis of biomarkers in soil groups [43]. LEfSe analysis was conducted on the website (http://huttenhower.sph.harvard.edu/galaxy) with factorial 
Kruskal-Wallis test among classes $<0.05$ and the threshold on the logarithmic LDA score $>3.5$. The metabolic functional features of bacterial communities were predicted by PICRUSt10 program in the context of the COG database [44].

\section{Qpcr Analysis Of Predominant Bacterial Community}

The main bacterial phyla ( $a$-Proteobacteria, $\beta$-Proteobacteria, Actinobacteria, Chloroflexi, Acidobacteria, and Cyanobacteria) and beneficial genus (Rhizobium) was measured by qPCR [31, 45]. qPCR analysis was performed by Bio-Rad CFX96 (Bio-Rad, Hercules, CA, USA) with the following reaction mixture: $0.5 \mu \mathrm{L}$ of primers $(10 \mu \mathrm{M}), 7.5 \mu \mathrm{L}$ ChamQ SYBR Color qPCR Master Mix (Q411, Vazyme, Nanjing, China), and template DNA. Primers are listed in Table S3.

\section{Statistical analysis}

All experiments were performed three times. PCA, PCoA, and hierarchical clustering analysis based on Bray-Curtis distances were performed to assess beta diversity patterns. Error bars in each graph of qPCR assays indicate the mean values \pm SEM of replicates. Statistically significant differences between measurements were determined using one-way ANOVA ( $P<0.05$; LSD and Duncan test) in Statistical Product and Service Solutions Statistics software (SPSS 24; IBM, Armonk, NY, USA) as the previous study [46].

\section{Abbreviations}

$\mathrm{CB}$

control bulk soil; COG:cluster of orthologous groups; CRBR:controlled rhizosphere soil of resistant biggrain peanut Huayu25; CRSR:controlled rhizosphere soil of resistant small-grain peanut Luhua12; CSBR:controlled rhizosphere soil of susceptible big-grain peanut Huayu33; CSSR:controlled rhizosphere soil of susceptible small-grain peanut Huayu20; LEfSe:Linear discriminant analysis (LDA) effect size; OTU:Operational taxonomic units; PCA:principal component analysis; PCoA:principal co-ordinates analysis; PGPRs:plant growth promoting rhizobacteria; qPCR:quantitative PCR; SRBR:salt-treated rhizosphere soil of resistant big-grain peanut Huayu25; SRSR:salt-treated rhizosphere soil of resistant small-grain peanut Luhua12; SSBR:salt-treated rhizosphere soil of susceptible big-grain peanut Huayu33; SSSR:salt-treated rhizosphere soil of susceptible small-grain peanut Huayu20

\section{Declarations}

\section{Ethics approval and consent to participate}

Not applicable.

\section{Consent for publication}


Not applicable, because our article used original illustrations.

\section{Availability of data and materials}

All relevant data and materials that support the findings of this study are available from the corresponding author upon request. The following information was supplied regarding data availability: Data is available at NCBI under the SRA accession SUB6650557 and bioproject PRJNA592826, database is accessible via the link https://www.ncbi.nlm.nih.gov/sra/PRJNA592826.

\section{Competing interests}

The authors declare that no competing interests exist.

\section{Funding}

This work was supported by the National Natural Science Foundation of China (Grant No. 31901574 and 31971856), the Modern Agricultural Industry Technical System of Shandong Province (Grant No. SDAIT04-06), and the Agricultural Scientific and the Technological Innovation Project of Shandong Academy of Agricultural Sciences (Grant No. CXGC2018B05).

\section{Author Contributions}

$Z Z$ and LD conceived and designed the study. YX, HD, SW and FQ performed theexperiments. YX, DC, GZ and GY performed sample collection, and soil chemical property analysis. YX, HD and LD analyzed data. YXand ZZ reviewed and edited the manuscript. All authors read and approvedthe manuscript.

\section{Acknowledgements}

Not applicable.

\section{Authors' information}

Yang Xu and Hong Ding contributed equally to this work.

Affiliations

${ }^{1}$ Shandong Peanut Research Institute, Shandong Academy of Agricultural Sciences, Qingdao, Shandong, 266100, P. R. China

${ }^{2}$ College of Agronomy, Xinjiang Agricultural University, Urumqi, Xinjiang, 830001, P. R. China

\section{References}

1. Asif MA, Zafar Y, Iqbal J, lqbal MM, Rashid U, Ali GM, Arif A, Nazir F. Enhanced expression of AtNHX1, in transgenic groundnut (Arachis hypogaea L.) improves salt and drought tolerence. Mol Biotechnol. 
2011;49(3):250-6.

2. Chakraborty K, Bhaduri D, Meena HN, Kalariya K. External potassium $(\mathrm{K}(+))$ application improves salinity tolerance by promoting $\mathrm{Na}(+)$-exclusion, $\mathrm{K}(+)$-accumulation and osmotic adjustment in contrasting peanut cultivars. Plant Physiol Biochem. 2016;103:143-53.

3. Damodharan K, Palaniyandi SA, Le B, Suh JW, Yang SH. Streptomyces sp. strain SK68, isolated from peanut rhizosphere, promotes growth and alleviates salt stress in tomato (Solanum lycopersicum cv. Micro-Tom). J Microbiol. 2018;56(10):753-9.

4. Deinlein U, Stephan AB, Horie T, Luo W, Xu G, Schroeder JI. Plant salt-tolerance mechanisms. Trends Plant Sci. 2014;19(6):371-9.

5. Mickelbart MV, Hasegawa PM, Bailey-Serres J. Genetic mechanisms of abiotic stress tolerance that translate to crop yield stability. Nat Rev Genet. 2015;16(4):237-51.

6. Yu Z, Duan X, Luo L, Dai S, Ding Z, Xia G. How Plant Hormones Mediate Salt Stress Responses. Trends Plant Sci 2020.

7. Ullah A, Akbar A, Luo Q, Khan AH, Manghwar H, Shaban M, Yang X: Microbiome Diversity in Cotton Rhizosphere Under Normal and Drought Conditions. Microb Ecol 2018.

8. Ullah A, Sun H, Yang X, Zhang X. Drought coping strategies in cotton: increased crop per drop. Plant Biotechnol J. 2017;15(3):271-84.

9. Xu Y, Zhang G, Ding H, Ci D, Dai L, Zhang Z. Influence of salt stress on the rhizosphere soil bacterial community structure and growth performance of groundnut (Arachis hypogaea L.). Int Microbiol 2020.

10. Lareen A, Burton F, Schafer P. Plant root-microbe communication in shaping root microbiomes. Plant Mol Biol. 2016;90(6):575-87.

11. Mendes R, Garbeva P, Raaijmakers JM. The rhizosphere microbiome: significance of plant beneficial, plant pathogenic, and human pathogenic microorganisms. FEMS Microbiol Rev. 2013;37(5):634-63.

12. Dennis PG, Miller AJ, Hirsch PR. Are root exudates more important than other sources of rhizodeposits in structuring rhizosphere bacterial communities? FEMS Microbiol Ecol. 2010;72(3):313-27.

13. Bai Y, Muller DB, Srinivas G, Garrido-Oter R, Potthoff E, Rott M, Dombrowski N, Munch PC, Spaepen S, Remus-Emsermann $\mathrm{M}$, et al. Functional overlap of the Arabidopsis leaf and root microbiota. Nature. 2015;528(7582):364-9.

14. Naylor D, DeGraaf S, Purdom E, Coleman-Derr D. Drought and host selection influence bacterial community dynamics in the grass root microbiome. ISME J. 2017;11(12):2691-704.

15. Evelin H, Kapoor R, Giri B. Arbuscular mycorrhizal fungi in alleviation of salt stress: a review. Ann Bot. 2009;104(7):1263-80.

16. Yang J, Kloepper JW, Ryu CM. Rhizosphere bacteria help plants tolerate abiotic stress. Trends Plant Sci. 2009;14(1):1-4. 
17. Geng LL, Shao GX, Raymond B, Wang ML, Sun XX, Shu CL, Zhang J. Subterranean infestation by Holotrichia parallela larvae is associated with changes in the peanut (Arachis hypogaea L.) rhizosphere microbiome. Microbiol Res. 2018;211:13-20.

18. Finkel OM, Castrillo G, Herrera Paredes S, Salas Gonzalez I, Dangl JL. Understanding and exploiting plant beneficial microbes. Curr Opin Plant Biol. 2017;38:155-63.

19. D'Auria JC, Gershenzon J. The secondary metabolism of Arabidopsis thaliana: growing like a weed. Curr Opin Plant Biol. 2005;8(3):308-16.

20. Dixon RA. Natural products and plant disease resistance. Nature. 2001;411(6839):843-7.

21. Qiao Q, Wang F, Zhang J, Chen Y, Zhang C, Liu G, Zhang H, Ma C, Zhang J. The Variation in the Rhizosphere Microbiome of Cotton with Soil Type, Genotype and Developmental Stage. Sci Rep. 2017;7(1):3940.

22. Gaiero JR, McCall CA, Thompson KA, Day NJ, Best AS, Dunfield KE. Inside the root microbiome: bacterial root endophytes and plant growth promotion. Am J Bot. 2013;100(9):1738-50.

23. Dai L, Zhang G, Yu Z, Ding H, Xu Y, Zhang Z. Effect of Drought Stress and Developmental Stages on Microbial Community Structure and Diversity in Peanut Rhizosphere Soil. Int J Mol Sci 2019, 20(9).

24. Bulgarelli D, Garrido-Oter R, Munch PC, Weiman A, Droge J, Pan Y, McHardy AC, Schulze-Lefert P. Structure and function of the bacterial root microbiota in wild and domesticated barley. Cell Host Microbe. 2015;17(3):392-403.

25. Lundberg DS, Lebeis SL, Paredes SH, Yourstone S, Gehring J, Malfatti S, Tremblay J, Engelbrektson A, Kunin V, Del Rio TG, et al. Defining the core Arabidopsis thaliana root microbiome. Nature. 2012;488(7409):86-90.

26. Apte SK, Reddy BR, Thomas J. Relationship between Sodium Influx and Salt Tolerance of NitrogenFixing Cyanobacteria. Appl Environ Microbiol. 1987;53(8):1934-9.

27. Singh $\mathrm{H}$. Desiccation and radiation stress tolerance in cyanobacteria. J Basic Microbiol. 2018;58(10):813-26.

28. Yang Y, Wang N, Guo X, Zhang Y, Ye B. Comparative analysis of bacterial community structure in the rhizosphere of maize by high-throughput pyrosequencing. PLoS One. 2017;12(5):e0178425.

29. Kostka JE, Prakash O, Overholt WA, Green SJ, Freyer G, Canion A, Delgardio J, Norton N, Hazen TC, Huettel M. Hydrocarbon-degrading bacteria and the bacterial community response in gulf of Mexico beach sands impacted by the deepwater horizon oil spill. Appl Environ Microbiol. 2011;77(22):796274.

30. Adesemoye AO, Kloepper JW. Plant-microbes interactions in enhanced fertilizer-use efficiency. Appl Microbiol Biotechnol. 2009;85(1):1-12.

31. Li Q, Chen J, Wu L, Luo X, Li N, Arafat Y, Lin S, Lin W. Belowground Interactions Impact the Soil Bacterial Community, Soil Fertility, and Crop Yield in Maize/Peanut Intercropping Systems. Int J Mol Sci 2018, 19(2). 
32. Xiao E, Ning Z, Xiao T, Sun W, Qiu Y, Zhang Y, Chen J, Gou Z, Chen Y. Variation in rhizosphere microbiota correlates with edaphic factor in an abandoned antimony tailing dump. Environ Pollut. 2019;253:141-51.

33. Per TS, Khan NA, Reddy PS, Masood A, Hasanuzzaman M, Khan MIR, Anjum NA. Approaches in modulating proline metabolism in plants for salt and drought stress tolerance: Phytohormones, mineral nutrients and transgenics. Plant Physiol Biochem. 2017;115:126-40.

34. Miranda RS, Alvarez-Pizarro JC, Costa JH, Paula SO, Prisco JT, Gomes-Filho E. Putative role of glutamine in the activation of CBL/CIPK signalling pathways during salt stress in sorghum. Plant Signal Behav. 2017;12(8):e1361075.

35. Xu Y, Yu Z, Zhang S, Wu C, Yang G, Yan K, Zheng C, Huang J. CYSTM3 negatively regulates salt stress tolerance in Arabidopsis. Plant Mol Biol. 2019;99(4-5):395-406.

36. Yu Z, Xu Y, Zhu L, Zhang L, Liu L, Zhang D, Li D, Wu C, Huang J, Yang G, et al. The Brassicaceaespecific secreted peptides, STMPs, function in plant growth and pathogen defense. J Integr Plant Biol. 2020;62(4):403-20.

37. Caporaso JG, Kuczynski J, Stombaugh J, Bittinger K, Bushman FD, Costello EK, Fierer N, Pena AG, Goodrich JK, Gordon JI, et al. QIIME allows analysis of high-throughput community sequencing data. Nat Methods. 2010;7(5):335-6.

38. Edgar RC, Haas BJ, Clemente JC, Quince C, Knight R. UCHIME improves sensitivity and speed of chimera detection. Bioinformatics. 2011;27(16):2194-200.

39. Blaxter M, Mann J, Chapman T, Thomas F, Whitton C, Floyd R, Abebe E. Defining operational taxonomic units using DNA barcode data. Philos Trans R Soc Lond B Biol Sci. 2005;360(1462):1935-43.

40. Lu Y, Chen J, Zheng J, Hu G, Wang J, Huang C, Lou L, Wang X, Zeng Y. Mucosal adherent bacterial dysbiosis in patients with colorectal adenomas. Sci Rep. 2016;6:26337.

41. Bates ST, Clemente JC, Flores GE, Walters WA, Parfrey LW, Knight R, Fierer N. Global biogeography of highly diverse protistan communities in soil. ISME J. 2013;7(3):652-9.

42. Wang Y, Sheng HF, He Y, Wu JY, Jiang YX, Tam NF, Zhou HW. Comparison of the levels of bacterial diversity in freshwater, intertidal wetland, and marine sediments by using millions of illumina tags. Appl Environ Microbiol. 2012;78(23):8264-71.

43. Segata N, Izard J, Waldron L, Gevers D, Miropolsky L, Garrett WS, Huttenhower C. Metagenomic biomarker discovery and explanation. Genome Biol. 2011;12(6):R60.

44. Langille MG, Zaneveld J, Caporaso JG, McDonald D, Knights D, Reyes JA, Clemente JC, Burkepile DE, Vega Thurber RL, Knight R, et al. Predictive functional profiling of microbial communities using $16 \mathrm{~S}$ rRNA marker gene sequences. Nat Biotechnol. 2013;31(9):814-21.

45. Xu Y, Zhang D, Dai L, Ding H, Ci D, Qin F, Zhang G, Zhang Z. Influence of Salt Stress on Growth of Spermosphere Bacterial Communities in Different Peanut (Arachis hypogaea L.) Cultivars. Int J Mol Sci 2020, 21(6). 
46. Yu Z, Zhang D, Xu Y, Jin S, Zhang L, Zhang S, Yang G, Huang J, Yan K, Wu C, et al. CEPR2 phosphorylates and accelerates the degradation of PYR/PYLs in Arabidopsis. J Exp Bot. 2019;70(19):5457-69.

\section{Figures}
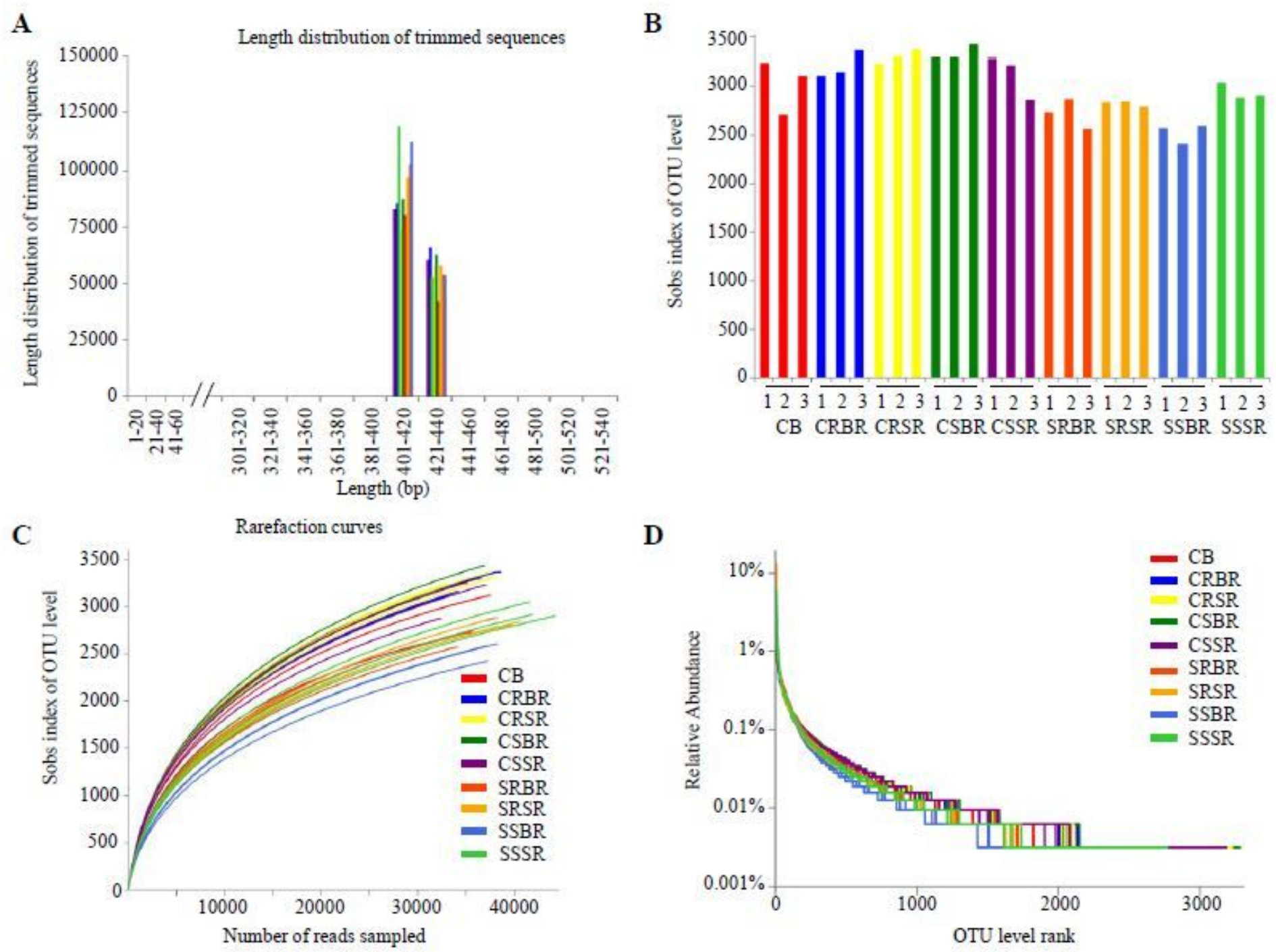

D

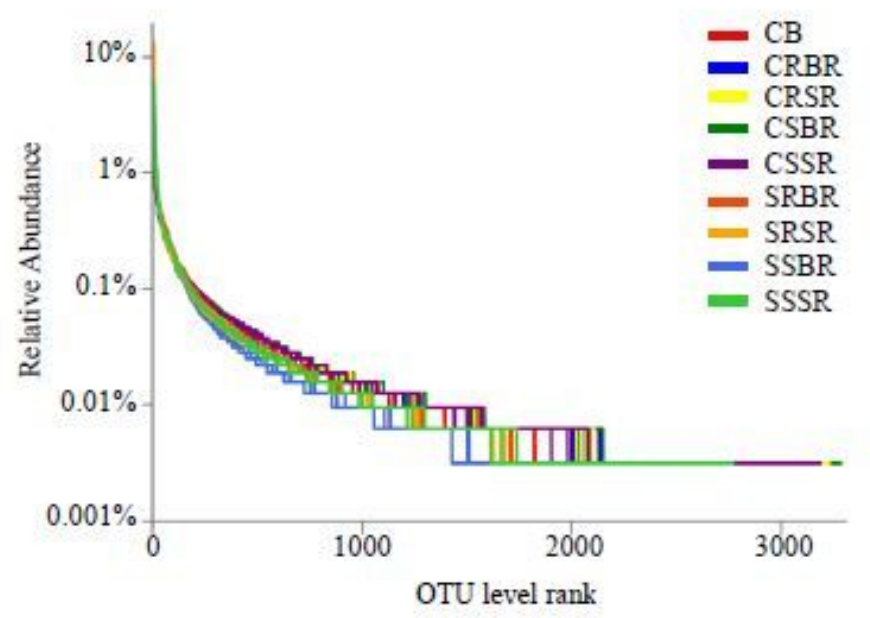

\section{Figure 1}

Alpha diversity analysis of peanut rhizosphere bacterial community richness and diversity (A) Length distribution of trimmed sequences in the peanut rhizosphere. (B) The operational taxonomic units (OTUs) levels of nine soil groups via sobs index analysis. CB, control bulk soil; CRBR, controlled rhizosphere soil of resistant big-grain peanut Huayu25; CRSR, controlled rhizosphere soil of resistant small-grain peanut Luhua12; CSBR, controlled rhizosphere soil of susceptible big-grain peanut Huayu33; CSSR, controlled rhizosphere soil of susceptible small-grain peanut Huayu20; SRBR, salt-treated rhizosphere soil of resistant big-grain peanut Huayu25; SRSR, salt-treated rhizosphere soil of resistant small-grain peanut Luhua12; SSBR, salt-treated rhizosphere soil of susceptible big-grain peanut Huayu33; SSSR, salt-treated 
rhizosphere soil of susceptible small-grain peanut Huayu20. (C) Rarefaction curve analysis showing the sequencing depth of peanut rhizosphere soils. (D) Rank abundance curve showing the relative species abundance and evenness. The length of polyline on the horizontal axis reflects the species abundance and the flatness of polyline reflects the species evenness.

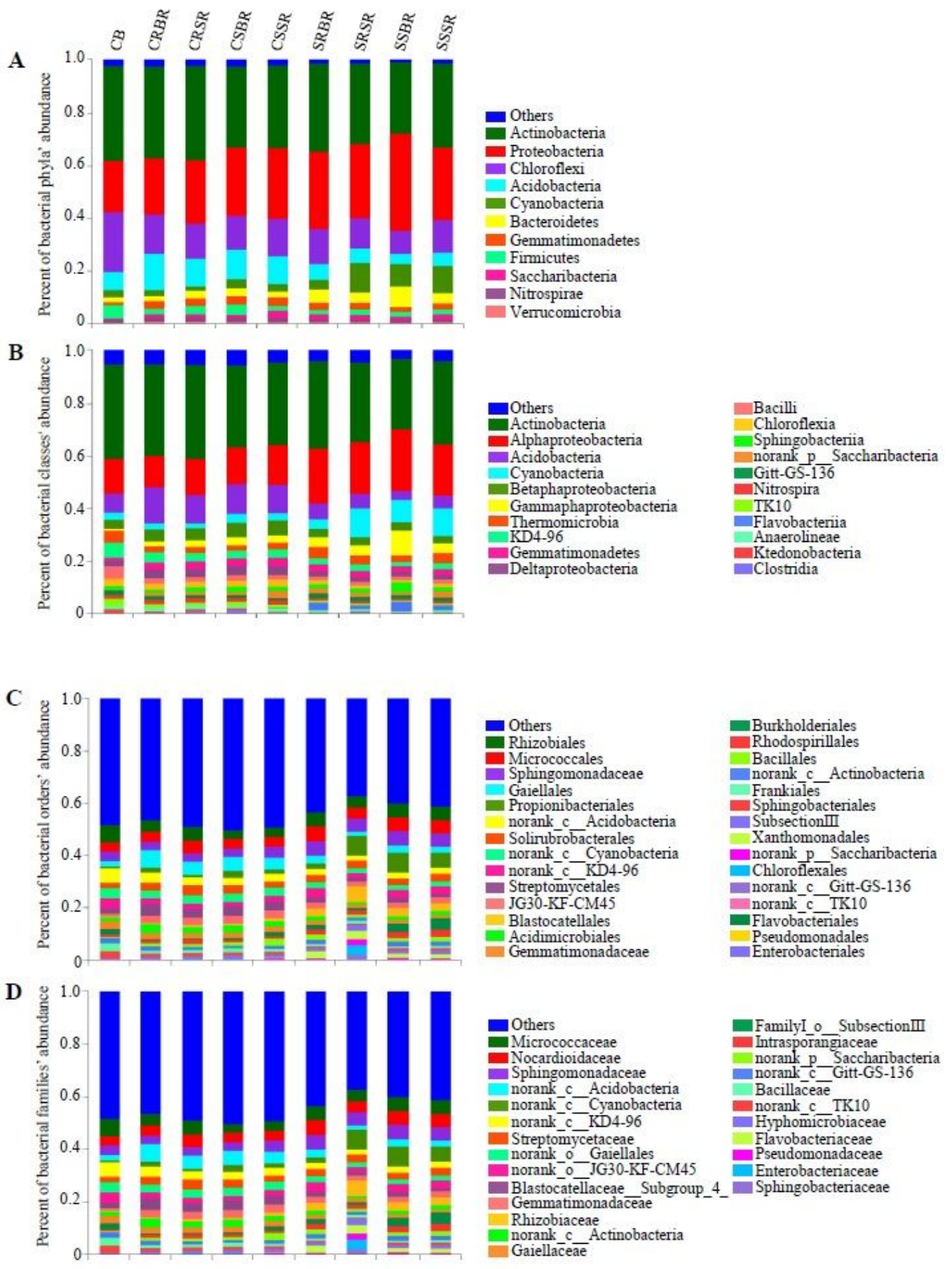

Figure 2 
Bacterial community structure of peanut rhizosphere soils The bacterial abundance distributions percent at the phylum level (A), at the class level (B), at the order level (C), and at the family level (D) in nine soil groups in peanut rhizosphere. The values are mean relative abundances across replicate samples. Both "norank" and "unclassified" in bacteria respect unidentified species obtained directly from the database via sequence alignment.
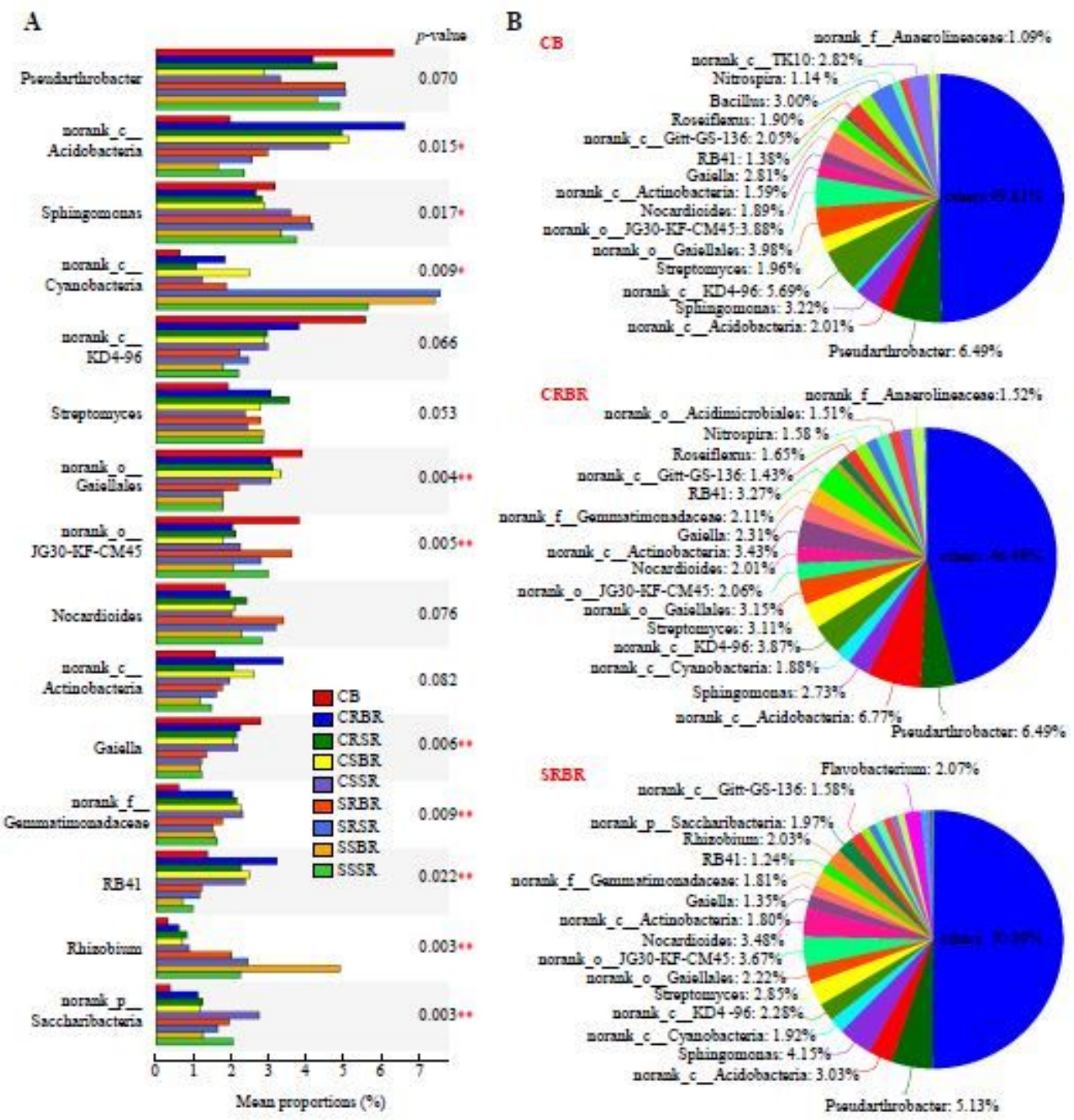

\section{Figure 3}

Bacterial abundance distributions in peanut rhizosphere at the genus level (A) The bacterial abundance distributions percent at the genus level in nine soil groups via the Kruskal-Wallis $\mathrm{H}$ test bar plot. The difference between proportions in 95\% confidence intervals is marked on the right. (B) Pie chart showing the abundance of bacterial genera in CB, CRBR, and SRBR using $16 \mathrm{~S}$ rRNA gene sequencing. 
A

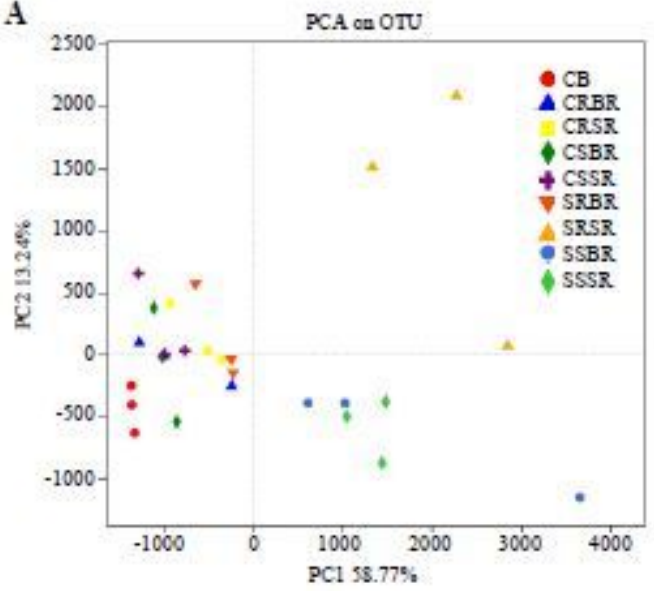

C

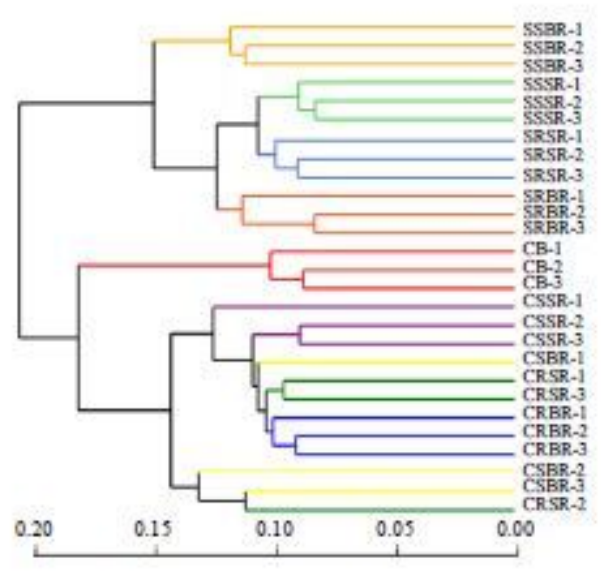

B

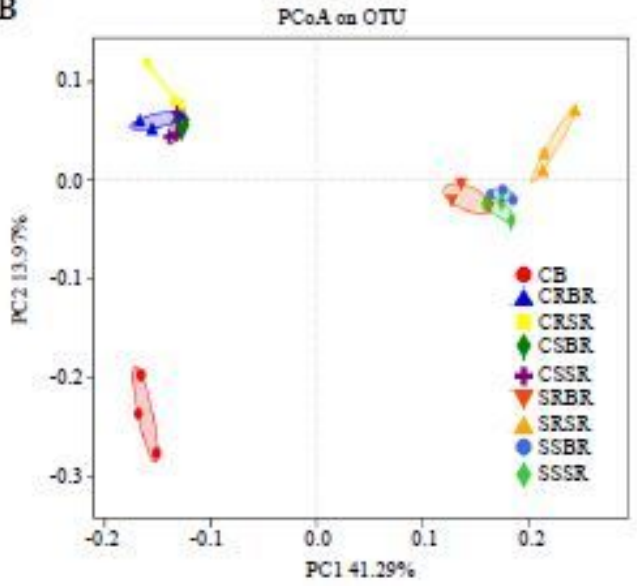

\section{Figure 4}

Beta diversity analysis (A) Two-dimensional ranking of the principal component analysis (PCA). (B) Multidimensional ranking of the principal co-ordinates analysis (PCOA). The same color points belong to the same soil group. (C) Hierarchical clustering analysis. Branch length indicates the similarity among the nine soil samples. 
A

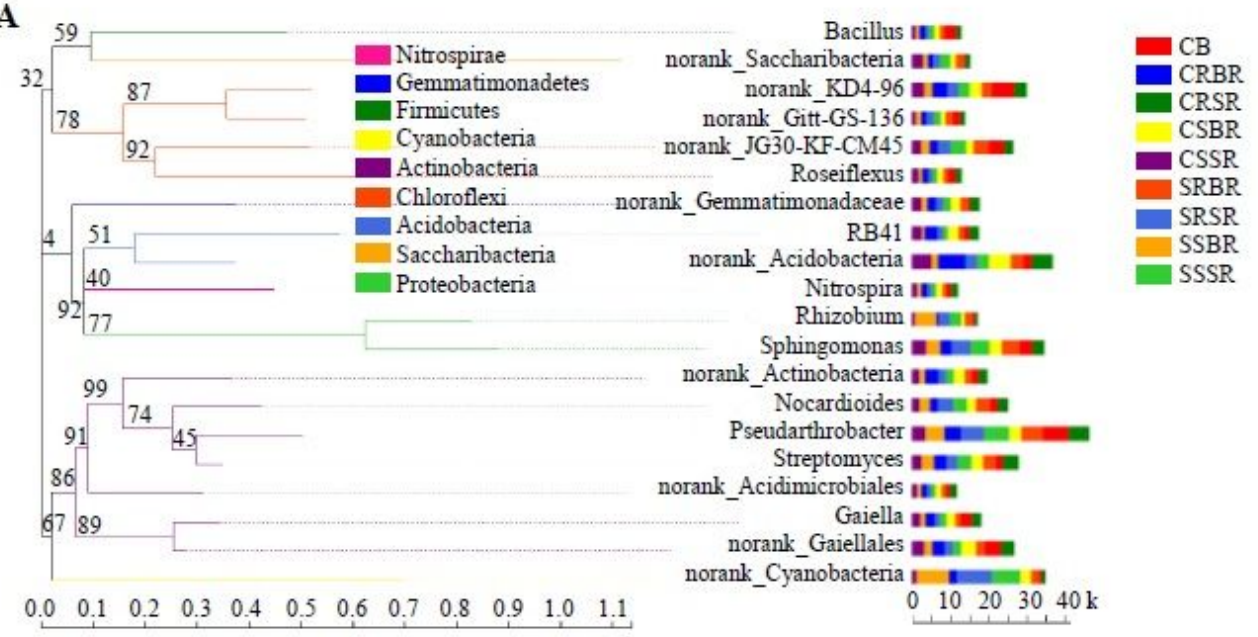

B

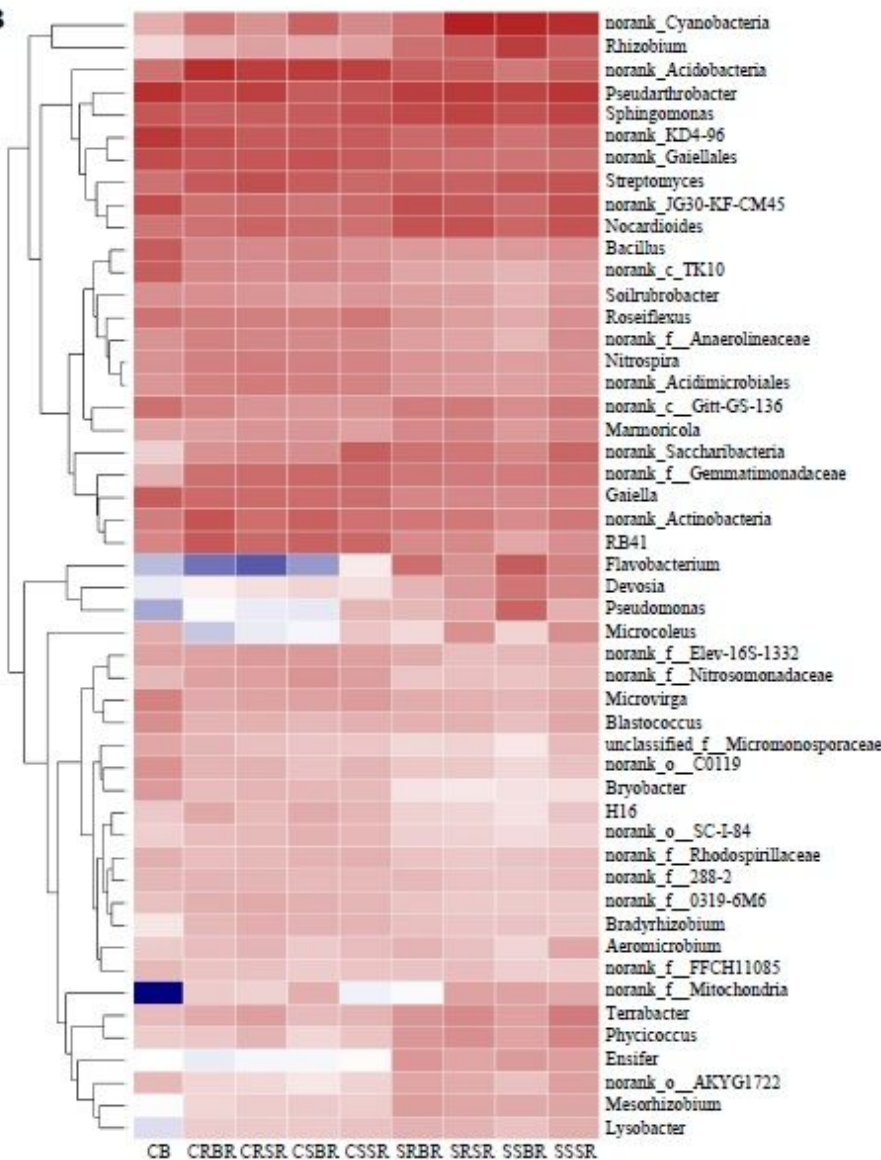

Figure 5

Phylogenetic tree and heat map (A) Phylogenetic tree showing the phylogenetic relationship of the bacterial community. Bootstrap values were obtained from 1000 repeated searches and shown on the nodes. The branch color on the left is the same as the color of the corresponding phylum, and the box color on the right is the same as the color of the corresponding soil. The box size on the right corresponds to the average relative abundance of the taxa in the corresponding soil. (B) The 50 most abundant genera 
were hierarchically clustered to generate heat maps and arranged in horizontal order according to the similarity among their components. The values are mean relative abundances across replicate samples. In the figure, red represents the more abundant genera in the corresponding sample, and blue represents the less abundant genera.
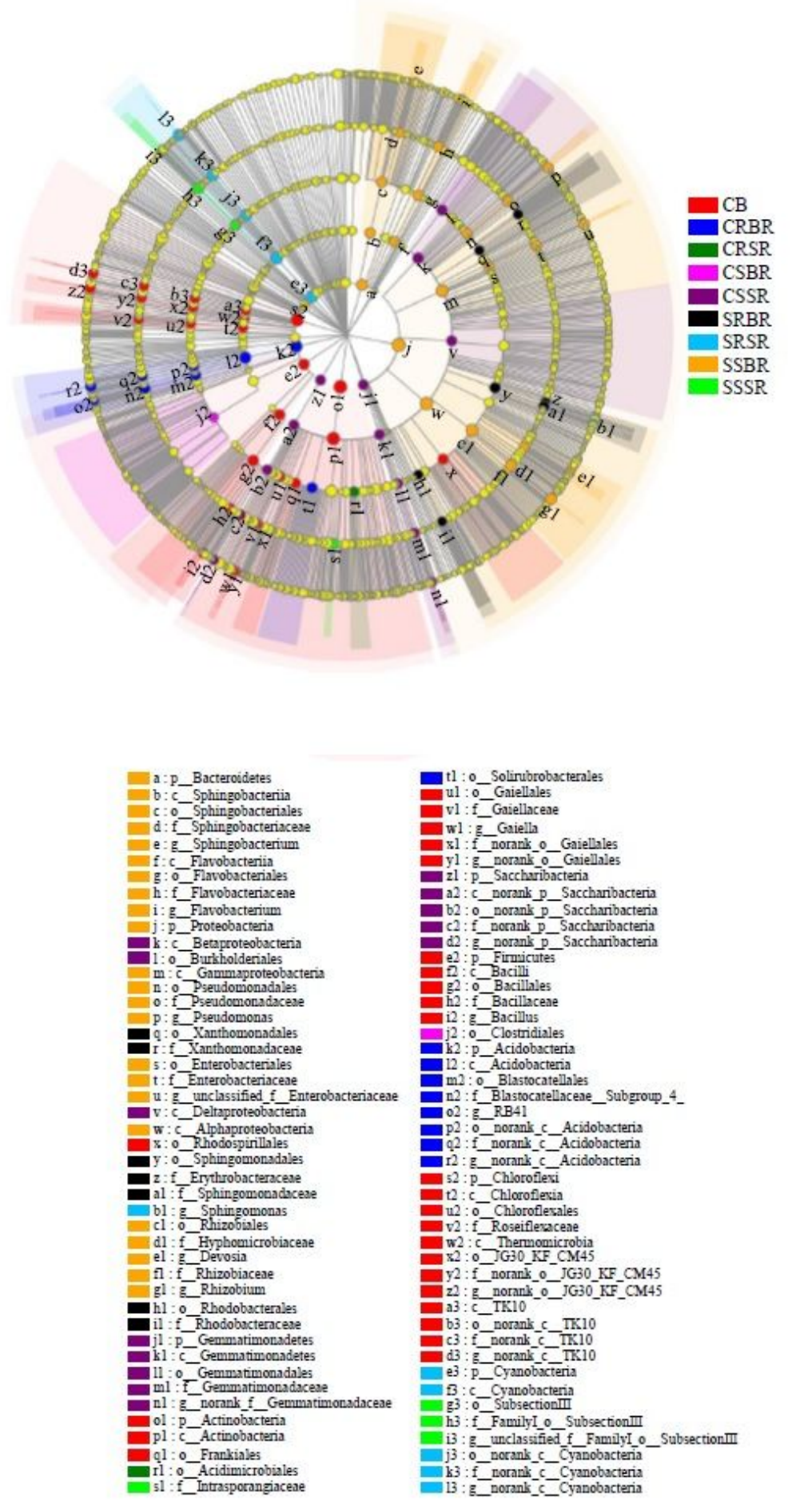

Figure 6 
Specific phylotypes of peanut rhizosphere modulated by salt stress in Cladogram Specific phylotypes of peanut rhizosphere bacterial communities with linear discriminant analysis (LDA) scores $>3.5$. From the inner circle to the outer circle indicates phylogenetic levels from phylum to genus. The circle size represents the bacterial abundance.

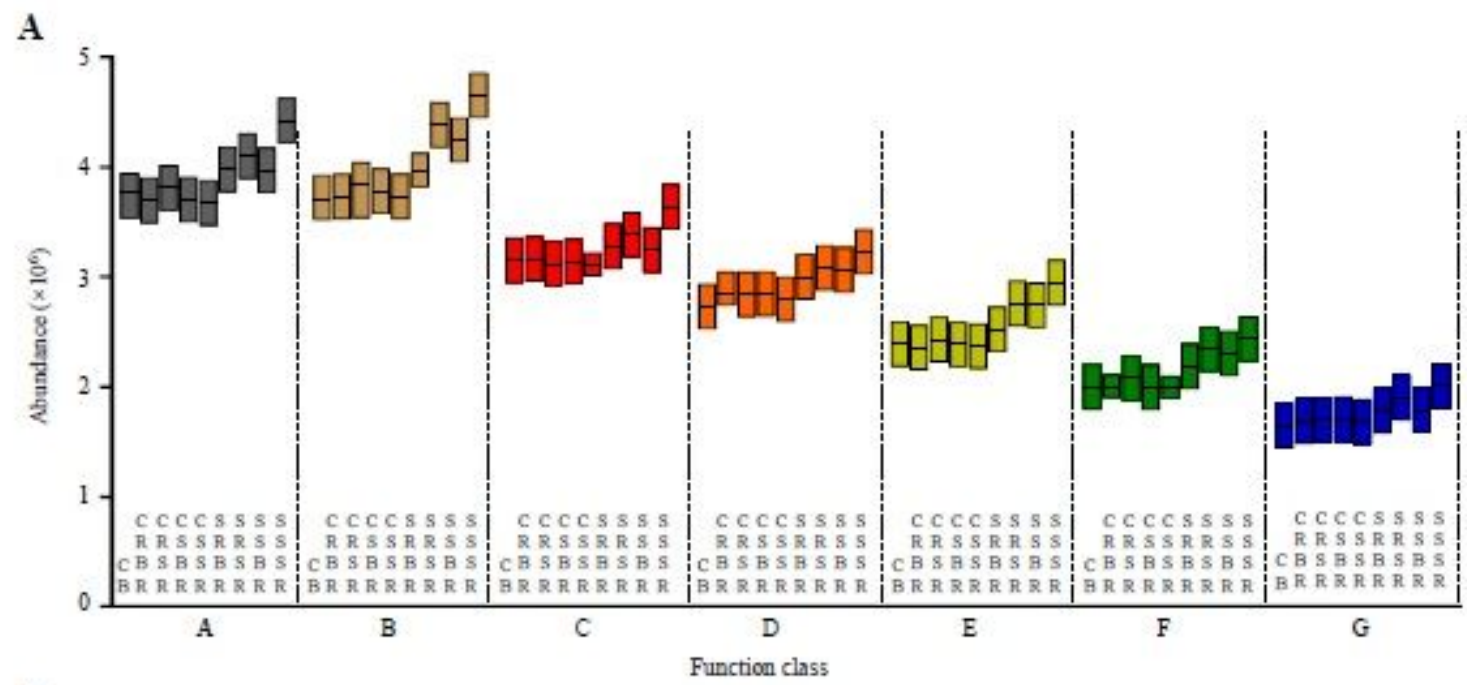

B

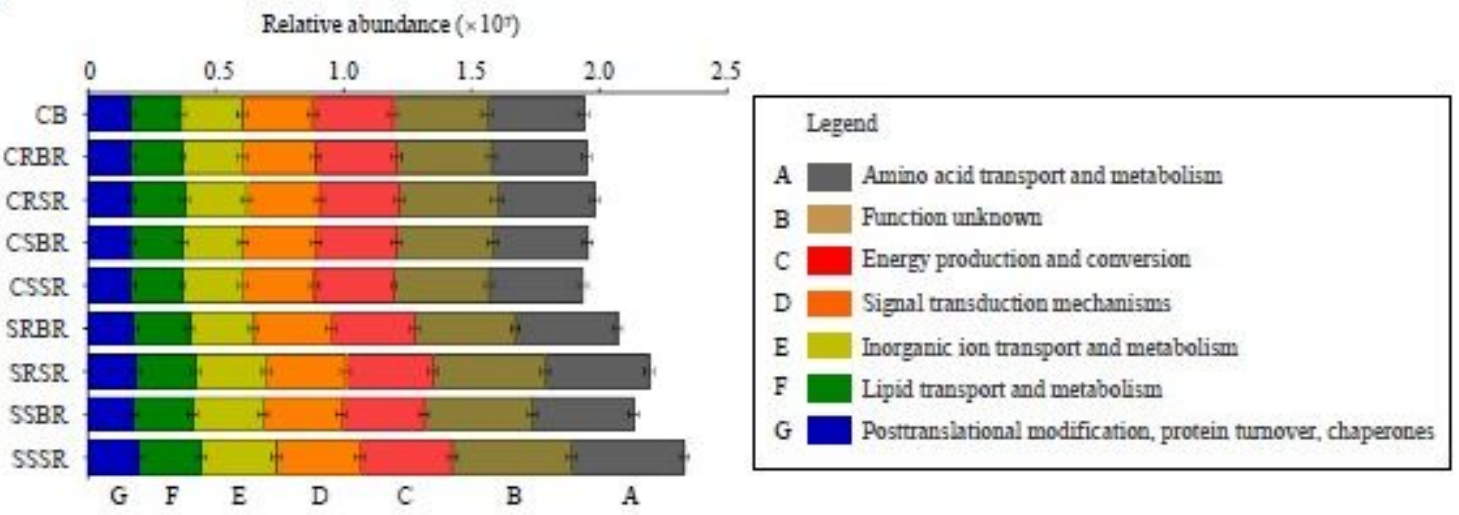

\section{Figure 7}

Metabolic functional features of nine rhizosphere soil (A) Box-plot showing the relative abundance of metabolic functional groups in nine soil groups (metabolic functional groups with a difference are shown here). (B) Bar chart showing the relative abundance of metabolic functional groups in nine soil groups (metabolic functional groups with a difference are shown here). The relative abundance is the average of the duplicated samples. 
A

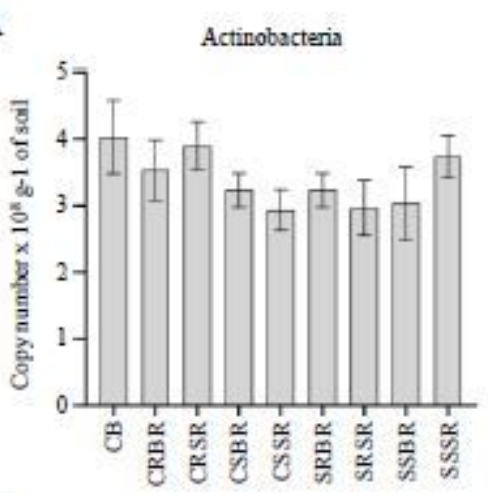

D
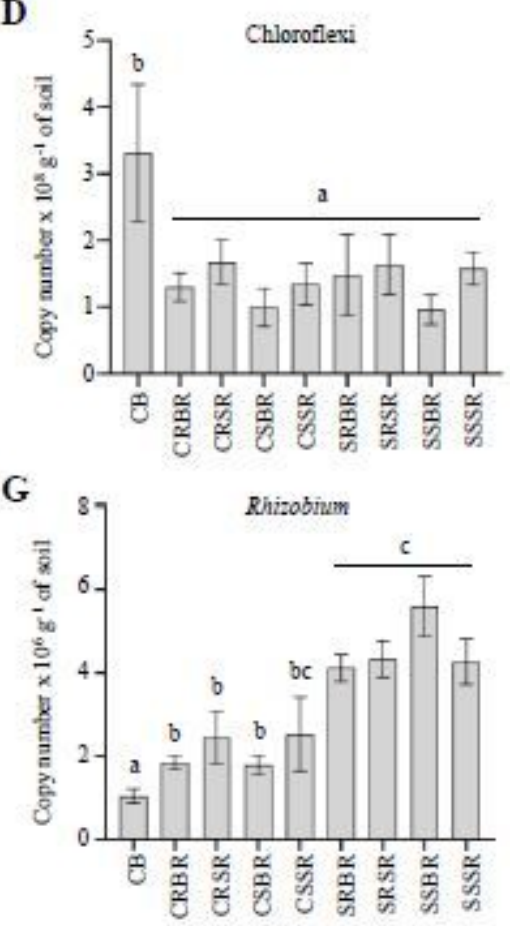

B

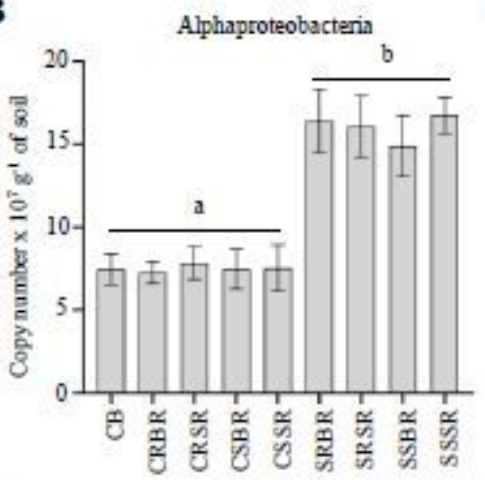

E

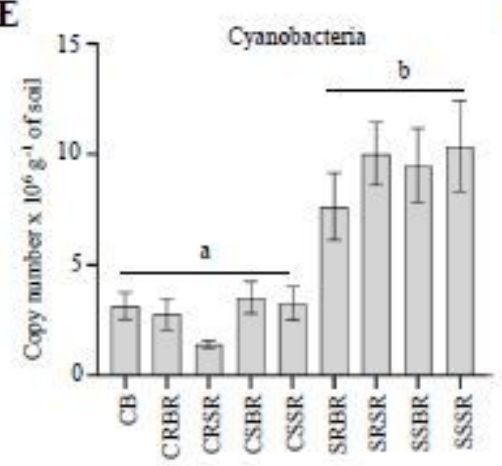

C

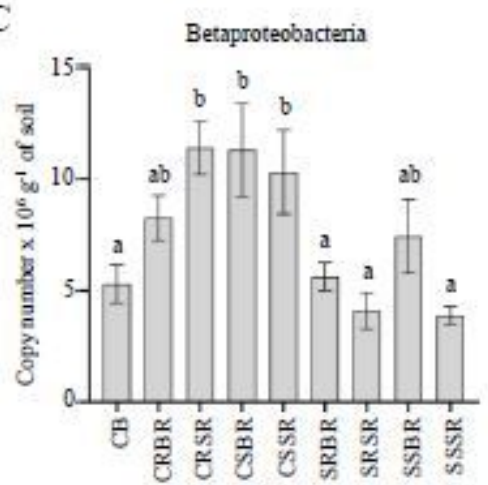

$\mathbf{F}$

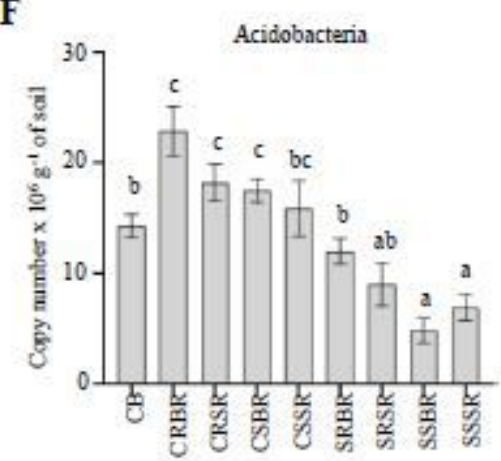

\section{Figure 8}

Quantification of genes involved in major bacterial communities under different peanut rhizosphere soils The genes of Actinobacteria (A); Alphaproteobacteria (B); Betaproteobacteria (C); Chloroflexi (D); Cyanobacteria (E); Acidobacteria (F); Rhizobium (G). Error bars indicate SEM (N=3), $p<0.05$. One-way ANOVA Duncan's test was used for statistical analysis. Statistical differences are indicated by lowercase letters and different letters represent different significance. These experiments were repeated three times.

\section{Supplementary Files}

This is a list of supplementary files associated with this preprint. Click to download.

- ExcelS1.xlsx

- Supplementarymaterials.pdf 